\title{
Hepatoblastoma with Multiple Tumors in a School-aged Child
}

\author{
Yugo Takaki ${ }^{1}$, Takahiro Yamashita ${ }^{2}$, Natsumi Kataoka ${ }^{1}$, Satomi Yokoyama ${ }^{1}$, Tadashi \\ Anan $^{2}$, Kimitoshi Nakamura ${ }^{2}$, Kazuhiko Yoshimoto ${ }^{1}$, Shintaro Hayashida ${ }^{2}$, Hirotoshi \\ Yamamoto $^{2}$, Hibi Taizo ${ }^{2}$, and Masahiro Migita ${ }^{1}$ \\ ${ }^{1}$ Japanese Red Cross Kumamoto Hospital \\ ${ }^{2}$ Kumamoto University Hospital
}

April 28, 2020

\begin{abstract}
The occurrence of hepatoblastoma is infrequent in children older than 5 years. Therefore, clinicians need to consider hepatoblastoma even in school-aged children or adolescents.
\end{abstract}

\section{Key Clinical Message}

Clinicians need to consider hepatoblastoma even in school-aged children or adolescents.

\section{Key Words}

Hepatoblastoma, Multiple tumors, School-aged children

\section{Text body}

A 10-year-old boy suffered from left hypochondrium pain for 3 days. He had a palpable stiff mass and tenderness in the upper abdomen. Laboratory examinations showed slightly elevated liver enzyme levels $(\mathrm{AST} / \mathrm{ALT}=74 / 38 \mathrm{U} / \mathrm{L} ; \mathrm{GGT}=38 \mathrm{U} / \mathrm{L})$, and markedly elevated serum alpha fetoprotein level $(88,000$ $\mathrm{ng} / \mathrm{mL}$ ). Abdominal ultrasonography revealed multiple tumors in the overall left and right liver parenchyma (Fig. 1). Abdominal computed tomography revealed that the liver contained PRETEXT III multiple tumors that were slightly enhanced in the arterial phase (Fig. 2A, B). He underwent extended left hepatectomy, and histopathology showed hepatoblastoma of the combined fetal and embryonal type. He received adjuvant chemotherapy and achieved complete remission for 10 months after chemotherapy without liver transplantation.

Hepatoblastoma is a rare primary malignant hepatic tumor that occurs during infancy and childhood, occurring predominantly in the first 2 years of life. The occurrence of hepatoblastoma is infrequent in children older than 5 years, and thus, the actual incidence could not be calculated (1). In most cases, hepatoblastoma is present as a solitary mass (2). Hepatoblastoma should always be considered even when the liver displays multiple tumors in school-aged children or adolescents.

Conflict of interest statement: The authors have no conflicts of interest to declare.

\section{Author Contributions}

All authors participated in the review of the manuscript. YT: drafted the manuscript. TY, NK, SY, TA, $\mathrm{KN}, \mathrm{KY}, \mathrm{SH}, \mathrm{HY}, \mathrm{TH}$ and MM read and approved the final manuscript.

\section{References}


1. Darbari A, Sabin KM, Shapiro CN, et al. Epidemiology of primary hepatic malignancies in U.S. children. Hepatology.2003;38:560-566.

2. Spector LG, Birch J. The epidemiology of hepatoblastoma. Pediatr Blood Cancer. 2012;59:776-779.

Figure legends

Figure 1.

Abdominal ultrasonography showing multiple tumors in the overall left and right liver parenchyma

Figure 2.

Computed tomography showing the liver with multiple tumors in axial image (A) and coronal image (B); white arrows, multiple tumors

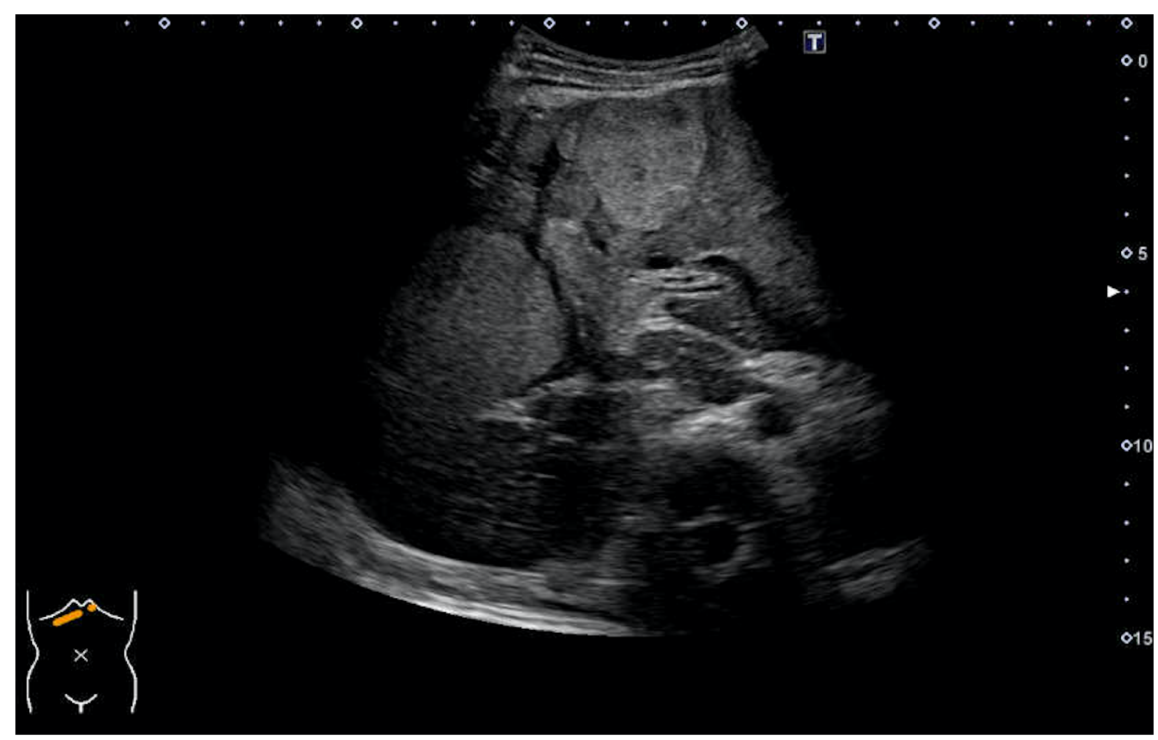

Figure 1
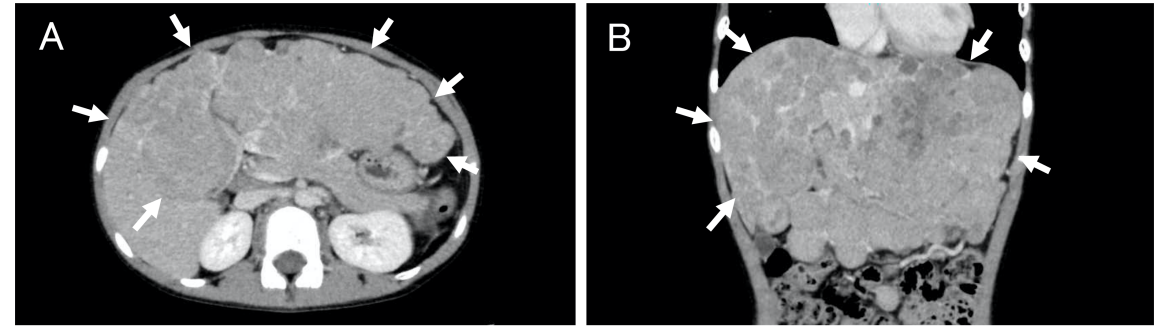

Figure 2 